\title{
Monte Carlo Simulation of Grain Growth Taking into Account the Influence of Temperature
}

\author{
Shigeaki OGIBAYASHI \\ Chiba Institute of Technology, 2-17-1 Tsudanuma, Narashino-shi, Chiba-ken 275-0016 Japan.
}

(Received on August 15, 2007; accepted on October 26, 2007)

\begin{abstract}
The influence of temperature on the grain growth behavior in Monte Carlo simulation has been studied based on the original Potts model proposed by Anderson et al. Grain growth kinetics in Monte Carlo simulation are represented in the non-dimensional form by non-dimensional grain radius and the ratio of Monte Carlo step to total orientation number $Q$. It has been revealed that the influence of temperature in Monte Carlo simulation could be mainly introduced through the correspondent relationship between Monte Carlo Step and real time, rather than through the transition probability of re-orientation attempts. A new equation for the relationship between Monte Carlo Step and real time has been derived based on the idea of the law of similitude in grain growth. The grain growth during cooling for the actual time-length system has been estimated using newly derived equation and the estimated grain size as a function of temperature showed quantitatively good agreement with the observed results reported in the literature when lattice constant in the calculation system as a characteristic length for conversion was set to be $0.015 \mathrm{~cm}$ which was a half of the initial grain diameter in the real system.
\end{abstract}

KEY WORDS: Monte Carlo simulation; computer simulation; grain growth; cooling rate; pinning effect; temperature factor; activation energy.

\section{Introduction}

Computer simulation ${ }^{1-6)}$ is widely used to clarify the influence of various factors on the crystal grain growth behavior, because the material properties of final steel product may greatly depend on the size of crystal grain. Computer simulation related to the crystal grain growth is divided into two stages, the $\gamma$ grain formation process during final stage of solidification and the $\gamma$ grain growth process during cooling after the complete solidification. Most of the previous studies on grain growth simulation, however, have dealt with the process at constant temperature, and the grain growth behavior during cooling from the state just after solidification to final product are not well understood.

Among various simulation models of the crystal grain growth such as the vertex model, ${ }^{4,5)}$ diffuse interface model, $\left.{ }^{6}\right)$ etc., the most promising is the Monte Carlo method proposed by Anderson et al. as the Potts model ${ }^{1,2)}$ and its modified models. ${ }^{7-10)}$ Although Monte Carlo method has presented successful results in simulating grain growth behavior such as the kinetics of grain growth under isothermal conditions, pinning effect by second phase particles, etc., there are few reports on the quantitative analysis of grain size and its dependency on temperature, mainly because the correspondence between the Monte Carlo Step and real time and the mechanism of the influence of temperature on the calculated results in the simulation are not well understood.
This paper presents studies on the influence of temperature in the Monte Carlo simulation and on the correspondence between the Monte Carlo Step and real time, from the viewpoint of the development of effective method in simulating grain growth behavior during cooling from the state just after solidification to final product. The influences of basic calculation conditions on the simulated results were also studied in order to confirm the validity of the present analysis.

\section{Simulation Method}

\subsection{Monte Carlo Simulation Method}

A continuum microstructure is mapped onto a discrete lattice. Each lattice site is assigned a positive integer between 1 and $Q$ corresponding to the orientation of the grain. The kinetics of grain boundary motion are simulated by employing a Monte Carlo technique, in which a lattice site is selected at random, and a new trial orientation is also chosen at random from one of the other $(Q-1)$ orientations. The change in energy caused by the change in orientation is then calculated according to Eq. (1), where $J$ is a positive constant in proportion to boundary energy per unit area, $s_{i}$ is the new trial orientation of the site, $s_{j}$ is the orientation of the nearest neighbor sites and $\delta_{i j}$ is the Kronecker delta. The sum is taken over all nearest neighbor sites. If the change in energy, $\Delta E$, is less than or equal to zero, the re-orientation is accepted. If the change in energy is greater than zero, the re-orientation is accepted with the transition 
probability, $P$, given by Eq. (2) where $k_{\mathrm{B}}$ is the Boltzman constant and $T$ is the temperature. This procedure is repeated $N_{\text {lattice }}$ times where $N_{\text {lattice }}$ is the total number of sites in the calculation system. The unit of time is defined as 1 Monte Carlo Step (MCS), which corresponds to $N_{\text {lattice }}$ re-orientation attempts.

$$
\begin{aligned}
& \Delta E=J \sum\left(\delta_{s_{i} s_{j}}-1\right) \ldots \ldots \ldots \ldots \\
P=\exp \left(-\Delta E / k_{\mathrm{B}} T\right) & (\Delta E>0) . \\
P=1 & (\Delta E \leq 0)
\end{aligned}
$$

\subsection{Condition of the Simulation}

Simulations were performed on 2-dimensional hexagonal lattice of size of $N=200^{2}$ as shown in Fig. 1. Each lattice was initially assigned one of $Q$ orientations randomly. In order to prevent the impingement of grain of the same orientation too frequently, a sufficiently large number of grain orientations $(Q)$ was chosen, typically $Q=64$. However, various values of $Q$ as $16,32,64,128,256$ were also tested in order to examine the influence of $Q$ on the kinetics of grain growth. Grain radius $R$ was calculated assuming one lattice area as a unit of the area.

Moreover, in order to confirm the validity of the calculation results by comparing them with the previous study results in the literature, ${ }^{1,9,11,12)}$ also studied were the behavior of a shrinking circular grain where $Q=2$ was chosen, and the pinning effect of second phase particles on grain growth behavior where $Q=-20$ was assigned at one or three lattice sites corresponding small and large particles. In case of simulation with the presence of second phase particles, the sites of the particles were selected at random and not allowed to switch during re-orientation attempts. The particle/matrix interfacial energy was assumed to be the same as the matrix grain boundary energy, $J$. The particle concentration was defined as the number of sites for particles divided by total number of the sites in the calculation, $N_{\text {lattice }}$.

In most of the calculation except for studying the influence of temperature, the transition probability given by Eq. (2) was assumed to be zero when the change in energy associated with the re-orientation was greater than zero.

In order to examine the influence of the temperature, the influence of $J / k_{\mathrm{B}} T$ on the average grain diameter and the transition probability for positive energy change were stud-

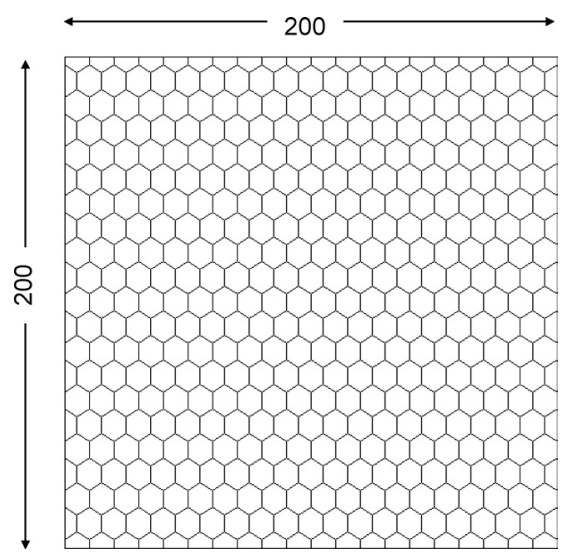

Fig. 1. 2-Dimensional hexagonal lattice used in the calculation. ied. In addition, the correspondence of $M C S$ and real time was discussed and a new method of converting calculated grain diameter to the grain diameter in real space has been derived. This new method was validated by comparing the calculated grain diameters during cooling with those calculated by Yoshida et al.'s equation ${ }^{14)}$ which were reportedly in good agreement with the observed results presented by Maehara et al. ${ }^{15)}$

\section{Simulation Results}

\subsection{Influence of the Number of Orientations on Grain Growth Kinetics}

Calculated microstructures for various values of the number of orientations, $Q$, at $1000 \mathrm{MCS}$ are shown in Fig. 2. It is obvious that grain diameters at constant $M C S$ become smaller with increasing $Q$. In the Monte Carlo simulation, grain radius is defined as shown in Eq. (3) and grain growth kinetics are expressed by using average grain radius $\bar{R}$. Calculated grain growth equations are summarized in Table 1.

$$
R=\sqrt{\frac{N S}{\pi}}=\sqrt{\frac{N}{\pi}} d
$$

where, $S$ : area of one lattice

$d=\sqrt{S}$ : lattice constant

$R$ : grain radius

$N$ : number of adjacent lattice sites having same orientation.

In Table 1, grain growth equations are expressed in the form of $\bar{R} / d=k(M C S)^{n}$ or $\bar{R} / d=k(M C S / Q)^{n}$, where $\bar{R}$ is the

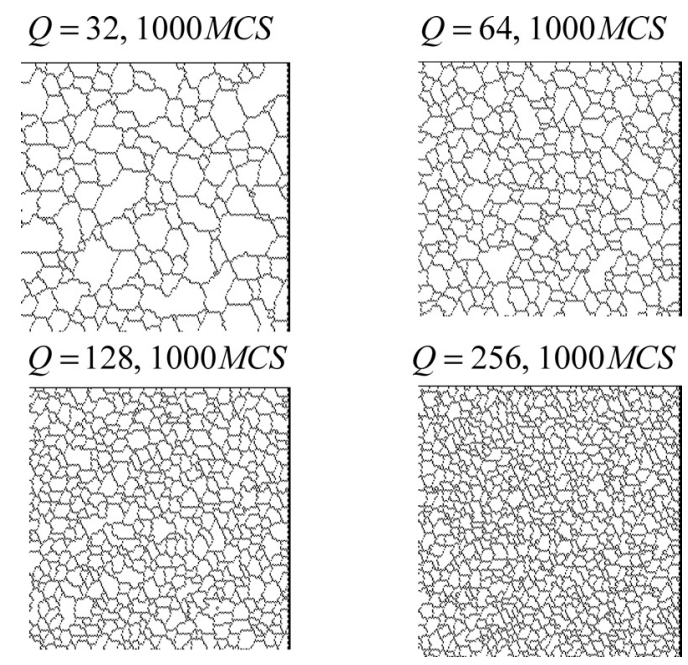

Fig. 2. Influence of $Q$ on grain structure at 1000 MCS.

Table 1. Grain growth equation calculated for various $Q$ values in the form of either $\bar{R} / d=k(M C S)^{n}$ or $\bar{R} / d=k(M C S / Q)^{n}$.

\begin{tabular}{|l|c|l|l|}
\hline $\begin{array}{l}\text { Trial } \\
\text { orientation }\end{array}$ & $Q$ & Grain growth Eq & Modified Eq \\
\hline \multirow{4}{*}{\begin{tabular}{l}
\multirow{4}{*}{$\begin{array}{l}\text { randum } \\
\text { rand }\end{array}$} \\
\cline { 2 - 4 }
\end{tabular}} & 16 & $0.36(\mathrm{MCS})^{0.40}$ & $1.08(\mathrm{MCS} / \mathrm{Q})^{0.40}$ \\
\cline { 2 - 4 } & 32 & $0.30(\mathrm{MCS})^{0.37}$ & $1.10(\mathrm{MCS} / \mathrm{Q})^{0.37}$ \\
\cline { 2 - 4 } & 128 & $0.28(\mathrm{MCS})^{0.34}$ & $1.15(\mathrm{MCS} / \mathrm{Q})^{0.34}$ \\
\cline { 2 - 4 } & 256 & $0.19(\mathrm{MCS})^{0.33}$ & $1.17(\mathrm{MCS} / \mathrm{Q})^{0.33}$ \\
\hline
\end{tabular}


average grain radius and $d$ is the lattice constant in the calculation system defined by the square root of the one lattice area as given by Eq. (3). As shown in Table 1, when grain radius is expressed as a function of $M C S$, coefficient $k$ decreases with increasing $Q$. When grain radius is expressed as a function of $M C S / Q$, on the other hand, coefficient $k$ does not depend on $Q$ when $Q$ is greater than 64 and the following grain growth equation is obtained, where exponent is 0.33 .

$$
R / d=1.16(M C S / Q)^{0.33}
$$

Regression analysis for grain growth taking account of initial grain size was also performed for $Q=64$ and the following equation was obtained, where initial grain radius $\bar{R}_{0}$ in the calculation was defined as the radius corresponding to one lattice area. The relationships between the grain radius and $M C S / Q$ based on the calculation and regression equation are compared in Fig. 3.

$$
(\bar{R} / d)^{2.77}-\left(\bar{R}_{0} / d\right)^{2.77}=1.24(M C S / Q)
$$

The exponents of grain growth obtained by the regression analysis are $0.33-0.36$ as shown in Eqs. (4) and (5) which are smaller than 0.5 . As shown in Table 1 , the exponent increases with decreasing $Q$. As a limiting case where $Q=2$, a shrinking behavior of a circle has been simulated. The area of the circle decreases in proportion to $M C S$ as shown in Fig. 4 and the regression equation shown in Eq. (6) was obtained, where $S$ is the area of one lattice in the

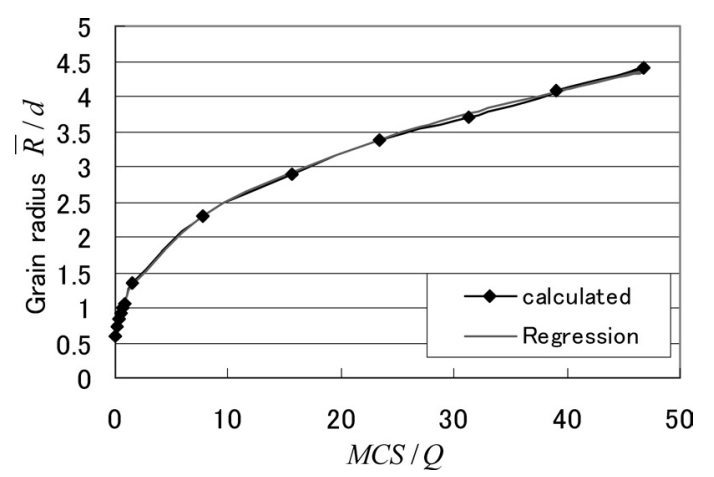

Fig. 3. Relationship between grain radius and $M C S / Q$, where grain radius is normalized by lattice constant in the calculation system and regression is performed taking into account initial grain size. calculation system. This means that the exponent of grain diameter in case of a shrinking circle is 0.5 which is in good agreement with that of the classical grain growth equation where the quadratic growth amount is proportional to time.

$$
A / S-A_{0} / S=-2.55 M C S .
$$

where, $A$ : area of a circle

$A_{0}$ : initial area of a circle

$S$ : one lattice are in the calculation system.

An example of the distribution of grain size normalized by average grain radius is given in Fig. 5 which shows nearly log-normal distribution but slightly differs as reported by Srolovitz et al., ${ }^{2)}$ in that the calculated distribution function is not symmetric in log-space and has an apparent upper cut-off and peaks more sharply than the lognormal distribution. It is also noted that the distribution of grain size is almost independent of MCS. This result suggests that the grain growth proceeds with the increase in diameter of large grains while small grains are diminishing. Close observation of Fig. 5 reveals that a small peak which arises in the frequency of the number of very small grain moves toward smaller grain size side with the increase in $M C S$. This tendency is considered to be caused because the diameter of relatively smaller grain decreases while that of larger grain increases in the process of grain growth.

\subsection{Pinning Effect of Second Phase Particles on Grain Growth}

It is reported in the literature ${ }^{11-13)}$ that the Monte Carlo simulation can also model grain growth in the presence of particle dispersion. In the present study, influences of the size and concentration of particles on grain growth behavior were analyzed, in order to confirm that the present calculation method is also effective in simulating the pinning

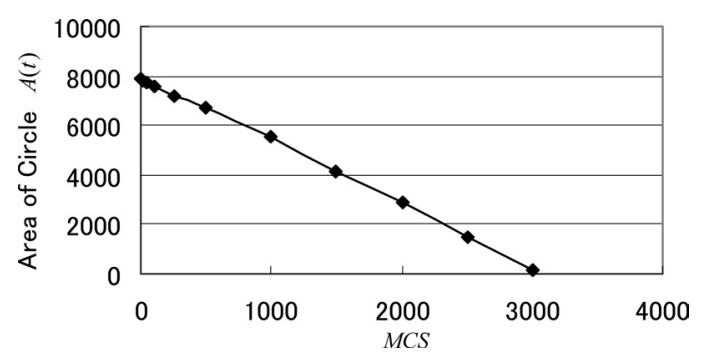

Fig. 4. Kinetics of a shrinking circular grain, showing that the area decreases linearly with time.

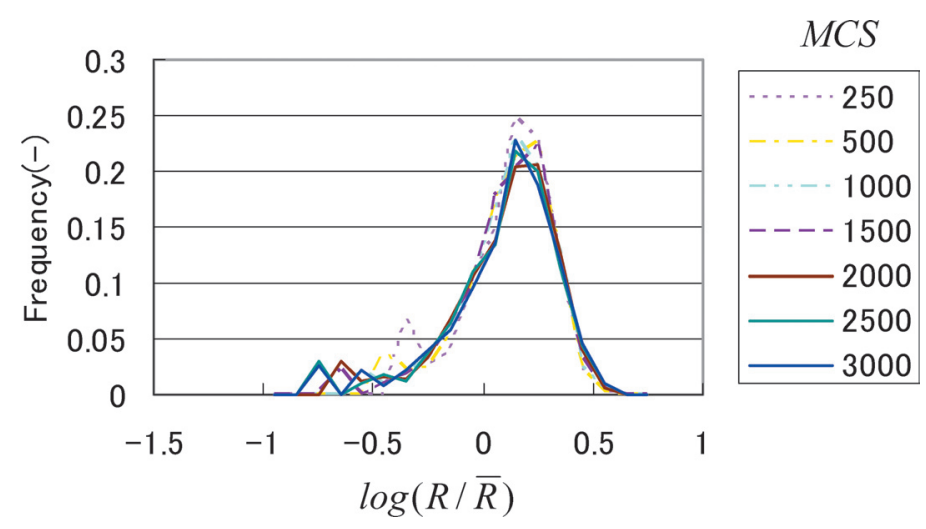

Fig. 5. Size distribution at various values of $M C S$ when $Q$ is 64 . 


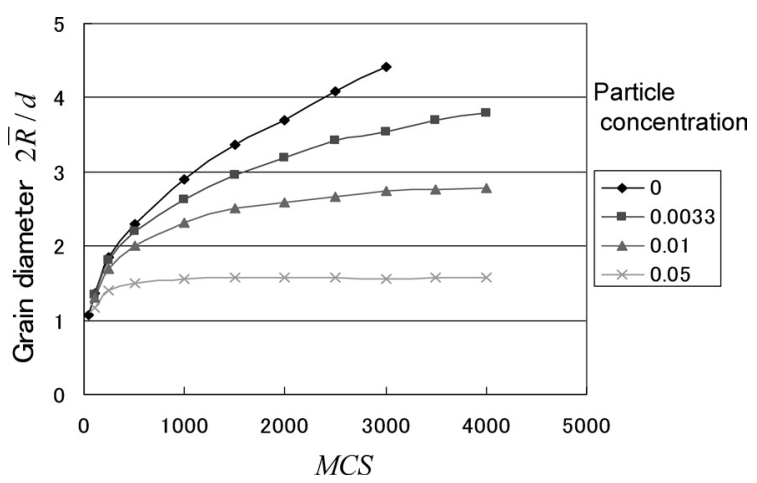

Fig. 6. Influence of particle concentration on grain growth, where grain diameter is normalized by lattice constant in the calculation system $(Q=64)$.

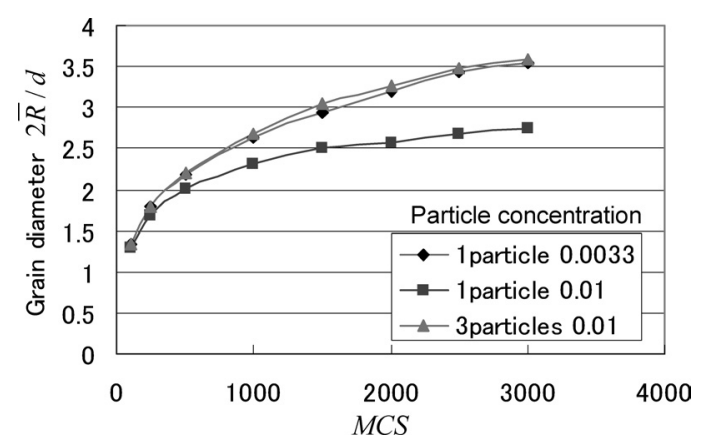

Fig. 7. Influence of particle size on pinning effect during grain growth, where grain diameter is normalized by lattice constant in the calculation system $(Q=64)$.

effect of second phase particles.

In case of the presence of particles, grain initially grows with increasing $M C S$, but it reaches a constant pinned grain size when $M C S$ is large enough. The pinned grain size is smaller when the particle concentration is larger, as shown in Fig. 6. It was also confirmed that, with the same particle concentration, the smaller the particle size is, the smaller the pinned grain size becomes as shown in Fig. 7. Moreover, the calculated results indicate that most of the particles locate on the grain boundary as shown in Fig. 8. The fraction of particles existing on the grain boundary decreases with increasing time as shown in Fig. 9 and exceeds $90 \%$, which became greater with increasing particle concentration.

\subsection{Influence of Temperature in the Monte Carlo Method}

One of the most serious problems of Monte Carlo simulation for grain growth is that the correspondence between Monte Carlo Step and real time and the mechanism of the influence of temperature on the calculated results in the simulation are not well understood and hence the method for quantitative analysis of grain size in real space taking into account its dependency on temperature has not been well developed.

In the algorithm of Monte Carlo simulation, there could be two possibilities in introducing the influence of temperature into the calculated results. First, the influence of temperature could be introduced in the calculation through the transition probability in re-orientation attempts. Second, it
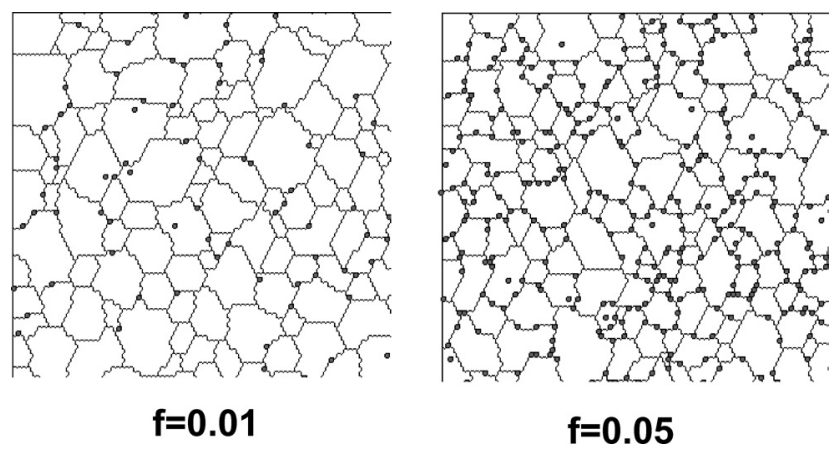

Fig. 8. Grain structure and location of particles represented by dark spots, showing that particles are likely to locate at grain boundary $(Q=64, M C S=3000, f$ : particle concentration).

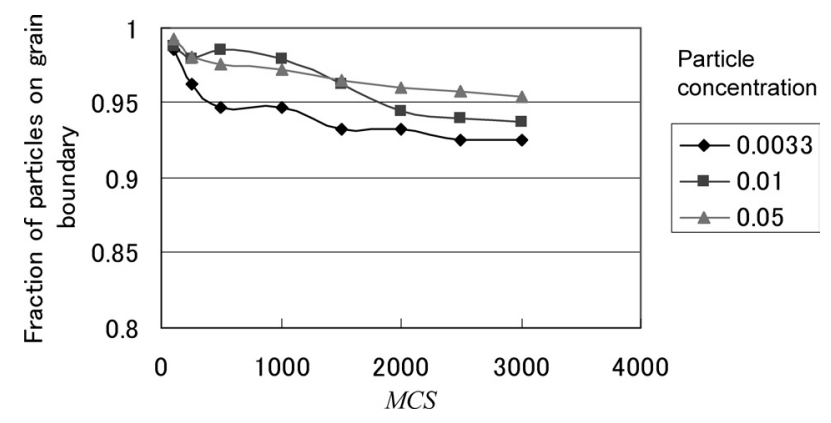

Fig. 9. Changes in fraction of particles on grain boundary with $M C S$ for various particle concentrations $(Q=64)$.

could be introduced through the correspondent relationship between the Monte Carlo Step and real time.

There are some reports on the relationship between the Monte Carlo Step and real time. ${ }^{8,14,15)}$ None of them, however, provide reliable evidence that the relationship has been well validated. Kobayashi et al. ${ }^{10)}$ explained the influence of temperature in Monte Carlo method from the viewpoint of transition probability. They ${ }^{10)}$ propose a new algorithm for transition probability of re-orientation, in which an activated state is introduced into the algorithm of defining transition probability and they point out that the influence of temperature can be well introduced into the simulation by their new algorithm. It seems reasonable to take into account the activation energy in the definition of transition probability, although the original Potts model reported by Anderson et al. ${ }^{1,2)}$ does not introduce activation energy in the transition probability. In the present study, however, the possibility of introducing the influence of temperature into the simulation algorithm is discussed based on the original Potts model from the viewpoint of transition probability and correspondent relationship of the Monte Carlo Step with real time.

\subsubsection{Influence of Temperature through Transition Proba- bility of Re-orientation}

As the transition probability in re-orientation attempts in case of the original Potts model is a function of $J / k_{\mathrm{B}} T$ as shown in Eq. (1), the influence of $J / k_{\mathrm{B}} T$ on grain size and transition probability was analyzed.

Grain structures for various values of $J / k_{\mathrm{B}} T$ at $2000 M C S$ with $Q=64$ are presented in Fig. 10, showing that fine structure arises near the grain boundary when $J / k_{\mathrm{B}} T$ 


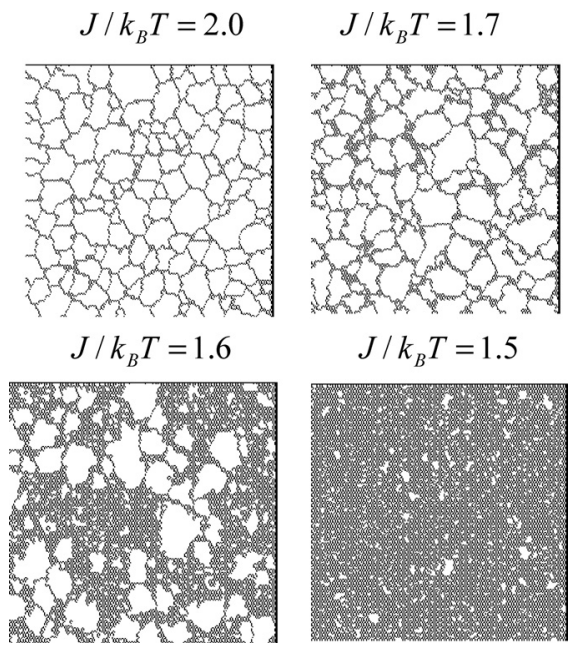

Fig. 10. Influence of $J / k_{\mathrm{B}} T$ on calculated grain structure $(Q=64$, $M C S=2000)$.

becomes less than 2.0 and it becomes a dominant structure when $J / k_{\mathrm{B}} T$ is 1.6 or below. This comes from the fact that the transition probability in re-orientation attempts for positive energy change given by Eq. (2) increases with increasing temperature.

The transition probability for positive energy change has been evaluated in the simulation by counting the frequency of accepting the re-orientation attempt when the energy change is positive. The calculated transition probability drastically increases with decreasing $J / k_{\mathrm{B}} T$ when $J / k_{\mathrm{B}} T$ is less than 1.6 as shown in Fig. 11. This tendency in transition probability is in good agreement with the dependency of grain structure on $J / k_{\mathrm{B}} T$ as shown in Fig. 10. The calculated results shown in Fig. 10 that fine structure that arises near the grain boundary becomes dominant when $J / k_{\mathrm{B}} T$ is less than 1.6 seems to be reasonable because the fluctuation of re-orientation at higher temperature sometime allows for the site to become a new small grain even though the associated energy change is positive and this probability becomes larger with increasing temperature. Therefore, the well known fact that grain growth rate increases with increasing temperature could not be explained by the factor of $J / k_{\mathrm{B}} T$ in the transition probability of the Potts model, as long as the transition probability given by Eq. (2) is assumed.

\subsubsection{Relationship between the Monte Carlo Step and Real Time}

It is well known that the grain growth proceeds due to the jumping of an atom from one side of the grain boundary to the other side with some transition probability which depends on the activation free energy. The atomic jump frequency, $v$, is related to macroscopic diffusion coefficient, $D$, as shown in Eq. (7) where $a$ is the lattice constant.

$$
v=\frac{6 D}{a^{2}}
$$

where, $D=D_{0} \exp \left(-\Delta E / k_{\mathrm{B}} T\right)$.

In the Monte Carlo method, grain growth kinetics are expressed as a function of $M C S / Q$ as shown in Fig. 3. If the probability of accepting re-orientation attempts is assumed to be proportional to $1 / Q, M C S / Q$ corresponds to the jump-

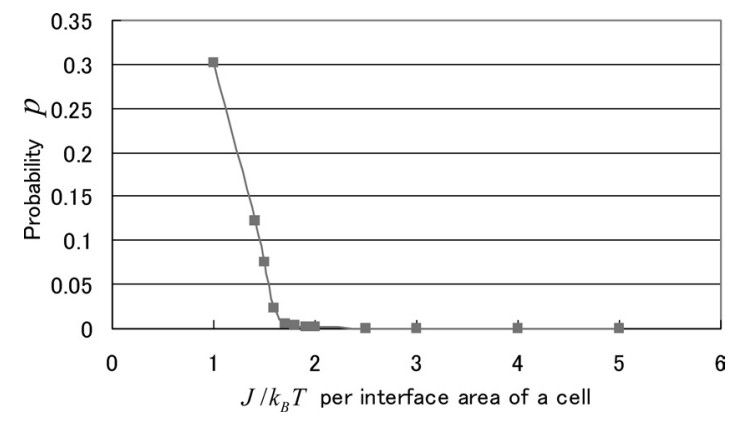

Fig. 11. Probability of orientation exchange as a function of $J / k_{\mathrm{B}} T$ when energy change is positive.

ing times during $M C S$. Therefore, $M C S / Q$ corresponds to $v t=6 D t / a_{2}$ which is the jumping times during $t$ in real system. The question is how the lattice constant should be defined in the relationship between $M C S / Q$ and $v t$, because the jumping distance for an atom is the lattice constant $a$ in real system, while in the simulation, the corresponding jumping distance due to one re-orientation attempt is the lattice constant $d$ in the calculation system.

According to classical grain growth theory, grain growth kinetics are represented by Eq. (8), where $\bar{R}$ is average grain radius, $\bar{R}_{0}$ is initial grain radius, $\sigma$ is interfacial energy, $V$ is molar volume, $R_{\mathrm{g}}$ is gas constant and $k_{0}$ is a non-dimensional parameter. When grain radius $R$ is represented by a non-dimensional radius $R^{\prime}=R / d$, where $d$ is a characteristic length for conversion, we can obtain Eq. (9) which shows that the law of similitude in grain growth is expressed by a non-dimensional factor $D t / d^{2}$.

where, $k_{0}=\sigma V / a R_{\mathrm{g}} T$,

$$
R^{2}-R_{0}^{2}=k_{0} D t
$$

$$
\bar{R}^{\prime 2}-\bar{R}_{0}{ }^{\prime 2}=k\left(D t / d^{2}\right)
$$

where, $R^{\prime}=R / d$.

Equation (9) for grain growth in the real system should be compared with Eq. (5) for grain growth obtained in the calculation system. Comparing both equations, it would be reasonable that the characteristic length for conversion from the calculation system to the real system can be set to be the lattice constant in the calculation system, and the relationship between $M C S$ and real time can be given by Eq. (10).

$$
M C S / Q=\frac{6 D t}{d^{2}}
$$

where, $d$ : lattice constant in the calculation system.

\subsection{Analysis of Grain Growth during Cooling and Comparison with the Data in the Real System}

An observed relationship between the grain diameter and temperature for grain growth during cooling with a constant cooling rate from the state just after solidification has been presented by Maehara et al. ${ }^{15)}$ where the initial grain diameter was $0.03 \mathrm{~cm}$. Yoshida et al. ${ }^{14)}$ has derived a prediction equation for grain growth during cooling based on the classical grain growth theory as shown in Eq. (11) and pointed out that the calculated grain size according to Eq. (11) showed good agreement with the observed result presented by Maehara et al. ${ }^{15)}$ as shown in Fig. 12. 


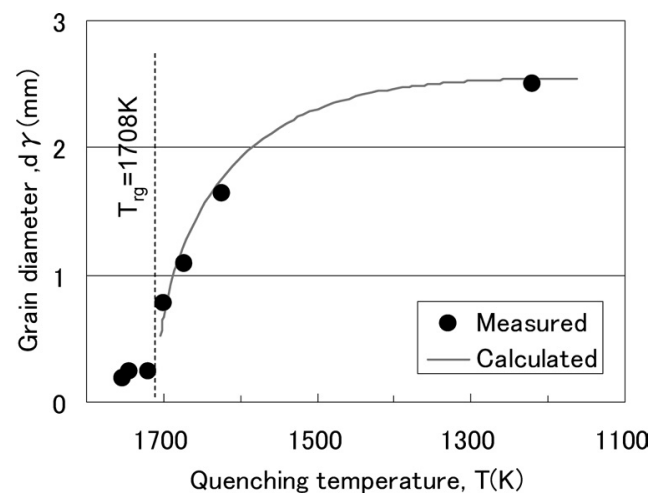

Fig. 12. Grain growth behavior during cooling with the cooling rate of $0.28 \mathrm{~K} / \mathrm{s}$, experimentally measured by Maehara et al. (dark spots) and calculated by Yoshida et al.'s equation based on classical grain growth model (solid line).

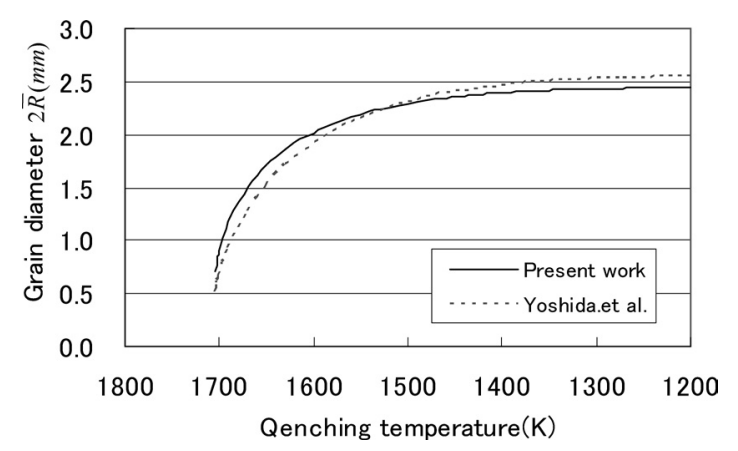

Fig. 13. Calculated grain diameter during cooling when lattice constant is $0.015 \mathrm{~cm}$, compared with Yoshida et al.'s equation based on classical grain growth model (cooling rate $=0.28 \mathrm{~K} / \mathrm{s}$, initial grain diameter $=0.03 \mathrm{~cm}$ ).

$$
\bar{R}^{2}-\bar{R}_{0}^{2}=\left(\sigma V / \lambda R_{\mathrm{g}} \dot{T}\right) \int_{T_{\mathrm{f}}}^{T_{\mathrm{rg}}}(D(T) / T) d T
$$

In the present study, relationship between $M C S$ and temperature during cooling has been derived as shown in Eq. (12) by transforming equation, Eq. (10). The grain growth during cooling has been analyzed by Monte Carlo method based on Eq. (12) by setting $D_{0}=1.6 \mathrm{~cm}^{2} / \mathrm{s}, Q_{\mathrm{gb}} / R_{\mathrm{g}}=$ $1.91 \times 10^{4} \mathrm{~K}$ and $\bar{R}_{0}=0.015 \mathrm{~cm}$ which are the same values used in Yoshida et al.'s analysis, ${ }^{14)}$ and calculated results have been compared with the predicted value calculated by Yoshida et al.'s equation ${ }^{14)}$ which can be used as an alternative for Maehara's observed result. ${ }^{15)}$ In the calculation, the lattice constant in the simulation system was changed between $0.01 \mathrm{~cm}$ and $0.1 \mathrm{~cm}$ and the grain diameter in the real system was calculated based on Eq. (12), which defines the relationship between $M C S / Q$ and temperature, and Eq. (5), which defines the relationship between grain radius and $M C S / Q$, using $T=0.28 \mathrm{~K} / \mathrm{s}, T_{0}=1708 \mathrm{~K}$ and $\bar{R}_{0}=0.015 \mathrm{~cm}$ which are the same condition as Maehara's data. ${ }^{14,15)}$

$$
M C S / Q=\int_{T_{\mathrm{f}}}^{T_{0}} \frac{6 D}{d^{2} \dot{T}} d T
$$

where, $T_{0}$ : initial temperature

$T_{\mathrm{f}}$ : temperature at $M C S$

$\dot{T}$ : cooling rate

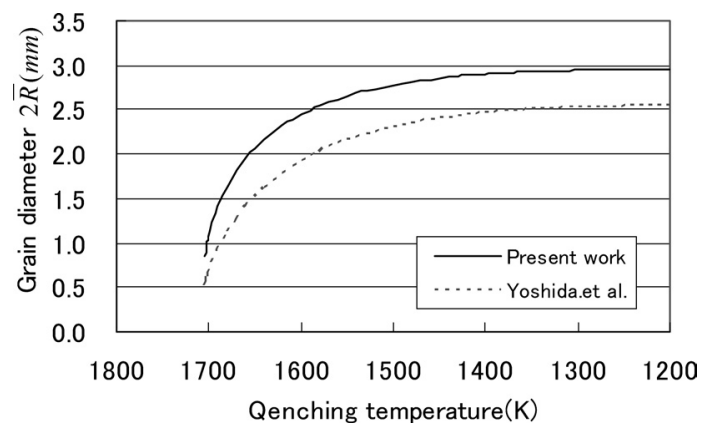

Fig. 14. Calculated grain diameter during cooling when lattice constant is $0.03 \mathrm{~cm}$, compared with Yoshida et al.'s equation based on classical grain growth model (cooling rate $=0.28 \mathrm{~K} / \mathrm{s}$, initial grain diameter $=0.03 \mathrm{~cm}$ ).

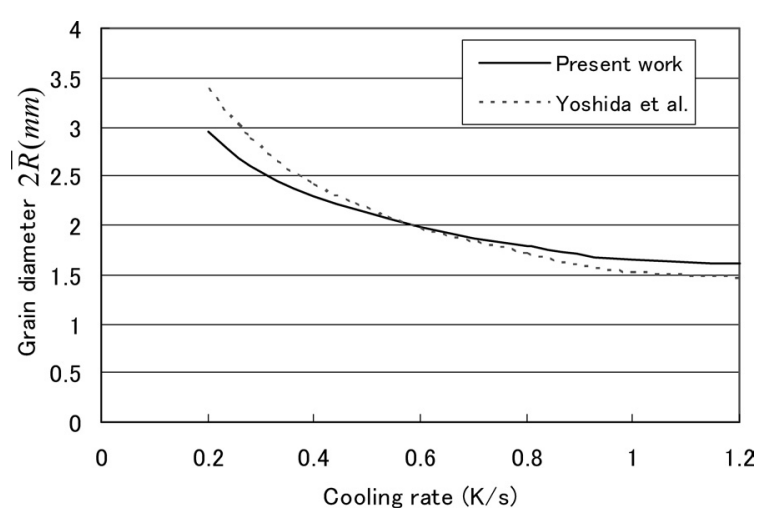

Fig. 15. Calculated grain diameter as a function of cooling rate at $1273 \mathrm{~K}$ when lattice constant is $0.015 \mathrm{~cm}$, compared with Yoshida et al.'s equation based on classical grain growth model.

$$
D=D_{0} \exp \left(-Q_{\mathrm{gb}} / R_{\mathrm{g}} T\right) .
$$

Calculated grain diameter during cooling compared with Yoshida et al.'s equation is shown in Fig. 13 as a function of quenching temperature. As shown in Fig. 13, the calculated relationship between the grain diameter and the quenching temperature is in good agreement with Yoshida et al.'s equation when the lattice constant as a characteristic value for conversion is set to be $0.015 \mathrm{~cm}$ which is half of the initial grain diameter. For comparison, the calculated grain diameter as a function of quenching temperature when the lattice constant is set to be $0.03 \mathrm{~cm}$ is shown in Fig. 14. It is obvious that the calculated grain diameter greatly differs with the Yoshida et al.'s equation when lattice constant is other than $0.015 \mathrm{~cm}$. Figure 15 shows the relationship between the grain diameter and the cooling rate at $1273 \mathrm{~K}$ when the lattice constant is again set to be $0.015 \mathrm{~cm}$ compared with Yoshida et al.'s equation. It is noted that the calculated result with the lattice constant of $0.015 \mathrm{~cm}$ is again in good agreement with Yoshida et al.'s equation.

Figures $\mathbf{1 6}$ and $\mathbf{1 7}$ show the influence of initial grain size and cooling rate on the grain diameter calculated by the Monte Carlo method of the present study and the one calculated by Yoshida et al.'s equation, respectively. It is noted in these results that the calculated relationship between the grain diameter and the temperature in the present study shows good agreement with Yoshida et al.'s equation for various initial grain sizes and cooling rates. It is also noted 


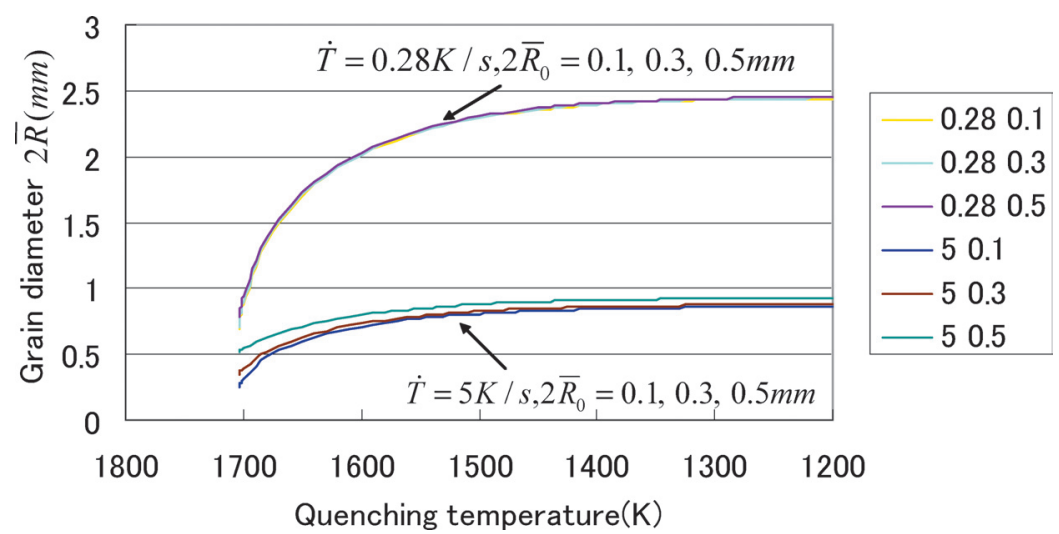

Fig. 16. Influence of initial grain size on grain diameter during cooling from $1704 \mathrm{~K}$ for cooling rate of $0.28 \mathrm{~K} / \mathrm{s}$ and $5 \mathrm{~K} / \mathrm{s}$ calculated in the present model with lattice constant of $0.015 \mathrm{~cm}$.

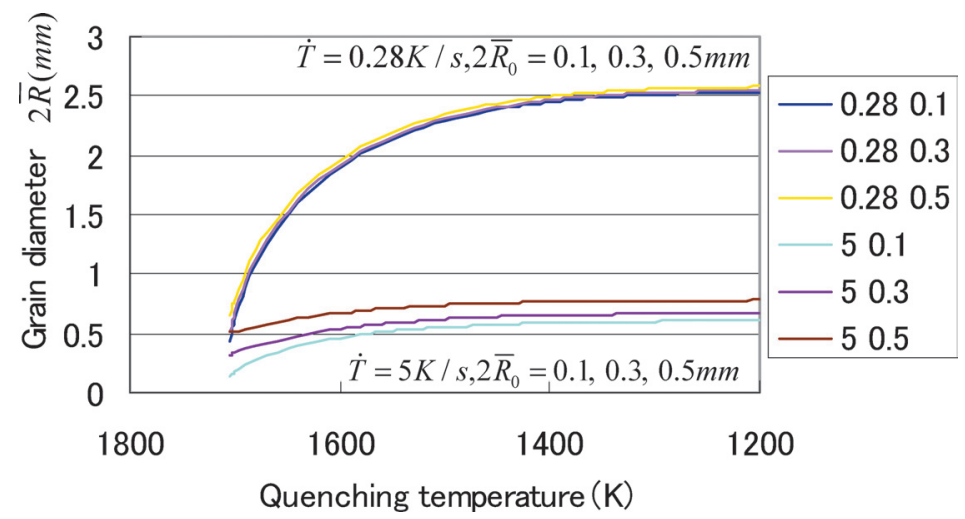

Fig. 17. Influence of initial grain size on grain diameter during cooling from $1704 \mathrm{~K}$ for cooling rate of $0.28 \mathrm{~K} / \mathrm{s}$ and $5 \mathrm{~K} / \mathrm{s}$ calculated by Yoshida et al.'s equation.

in Fig. 16 that the grain diameter at a cooled temperature is influenced by the initial grain size when the growth amount during cooling is relatively small because of large cooling rate $(5 \mathrm{~K} / \mathrm{s})$, although the influence of initial grain size is negligibly small when cooling rate is very small $(0.28 \mathrm{~K} / \mathrm{s})$.

\subsection{Discussions}

3.5.1. Mechanism of Grain Growth in Monte Carlo Simulation

In order to understand the basic mechanism of grain growth in the Monte Carlo method, the transition probability of orientation exchange has been examined. The transition probability is defined as the number of orientation-exchange sites per $M C S$, where re-orientation attempts were accepted, divided by the total number of lattice sites. It was revealed that the transition probability decreases with increasing time or grain radius during grain growth and is inversely proportional to $Q$ as shown in Fig. 18. Regression analysis revealed that the transition probability is quantitatively represented by Eq. (13)

$$
p=\frac{0.60}{Q}(\bar{R} / d)^{-3.49}
$$

If the transition probability at a lattice site is denoted by $p$, the number of orientation-exchange sites per MCS is represented by $N_{\text {lattice }} p$ where, $N_{\text {lattice }}$ is the total number of lattice sites in the calculation system. In addition, if $N$ stands for the average number of sites per grain, the average num-

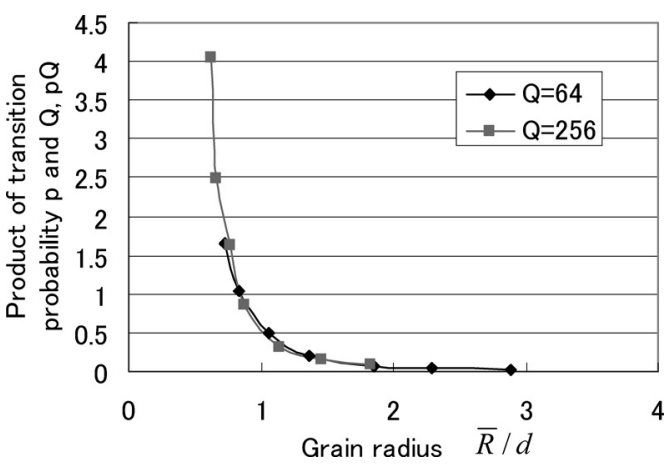

Fig. 18. Product of transition probability of orientation exchange $p$ and $Q, p Q$, as a function of grain radius normalized by latticed constant in the calculation system.

ber of grain is expressed by $N_{\text {lattice }} / \bar{N}$ and the number of orientation-exchange sites per $M C S$ per grain is expressed by $\bar{N} p$. In the normal grain growth, orientation-exchange occurs in the direction of grain growth. Therefore, the increased increment of $\bar{N}$ during $d(M C S)$, which is denoted by $d \bar{N}$, is equal to $\bar{N} p d(M C S)$. Thus, we can obtain Eqs. (14) and (15) which represent simplified grain growth model in Monte Carlo simulation.

$$
\begin{gathered}
\frac{d \bar{N}}{d(M C S)}=\bar{N} p \\
\frac{d \bar{R}}{d(M C S)}=\frac{1}{2} \bar{R} p
\end{gathered}
$$




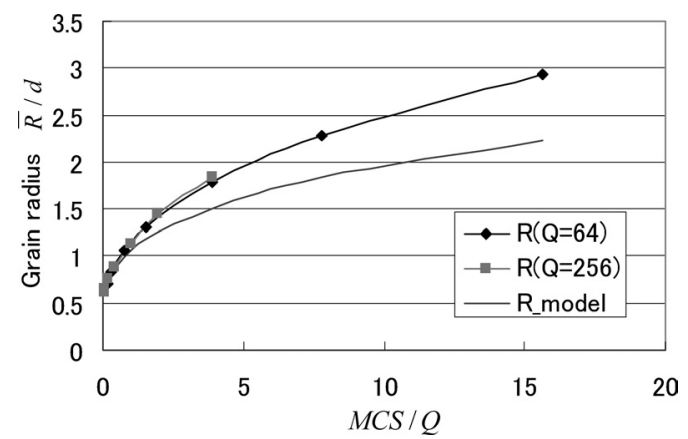

Fig. 19. Grain radius predicted by the model as a function of $M C S / Q$, compared with those calculated by Monte Carlo method for $Q=64$ and 256, where grain radius is normalized by lattice constant in the calculation system.

Solving Eq. (15) by substituting Eq. (13) for $p$, Eq. (16) is obtained. The average grain radius predicted by Eq. (16) is shown in Fig. 19 as a function of $M C S / Q$, in comparison with those calculated by Monte Carlo method for $Q=64$ and 256. Although the predicted average grain radius is a little smaller than the calculated radius, it seems to be concluded that the simplified grain growth model presented by Eq. (15) explains most of the grain growth behavior in Monte Carlo simulation. The discrepancy between the predicted radius and the calculated radius seems to be due to the influence of size distribution on the grain growth behavior.

$$
(\bar{R} / d)^{3.49}-\left(\bar{R}_{0} / d\right)^{3.49}=1.04(M C S / Q)
$$

\subsubsection{Influence of Temperature on Monte Carlo Method}

In the present study, the influence of temperature on the Monte Carlo method was discussed based on the original Potts model where transition probability is given by Eq. (2). As a result, the influence of temperature could not be explained by the factor of $J / k_{\mathrm{B}} T$ in the transition probability of the Potts model, as long as the transition probability given by Eq. (2) is assumed, and it was revealed that the influence of temperature can be well explained by introducing a new method of converting the Monte Carlo Step to real time.

Although the new method of converting the Monte Carlo Step to real time proposed in the present study has provided a successful result in explaining the actual grain growth behavior during cooling observed by Maehara et al., ${ }^{15}$ ) there are still some discrepancies remained for full understanding of the influence of temperature on grain growth in Monte Carlo simulation. Firstly, the present algorithm cannot explain the influence of interfacial energy on grain growth, because there is no factor of interfacial energy in Eqs. (10) and (12). The classical grain growth model presented by Yoshida et al. ${ }^{14)}$ on the other hand, includes interfacial energy as given by Eq. (11). In fact, it has been reported in the literature ${ }^{18)}$ that grain growth rate decreases by the addition of phosphorous, and Yoshida et al. ${ }^{18)}$ point out that one of the reasons for this influence is due to the decrease in interfacial energy by the addition of phosphorous. Secondly, the present algorithm assumes that the transition probability is expressed by Eq. (2), in which $\Delta E$ is the difference in interfacial energy between the nearest neighbors before and after the orientation exchange. This means that the present algorithm which is based on the original Potts model assumes to neglect activation energy. If the transition probability is assumed to be dependent on activation energy, influence of temperature could be explained to some extent by the dependency of transition probability on temperature, as pointed out by Kobayashi et al. ${ }^{10)}$

From these arguments, further study from the viewpoint of transition probability would be required for the full understanding of the influence of temperature on grain growth in Monte Carlo simulation, although the influence of temperature is considered to be mainly explained through the correspondent relationship of the Monte Carlo Step with real time.

\section{Conclusions}

Grain growth behavior has been analyzed using the Potts model of the Monte Carlo method proposed by Anderson et al. and it has been revealed that grain growth kinetics can be represented by the relationship between non-dimensional grain radius and $M C S / Q$. The validity of the present calculation has been confirmed from the viewpoint of the pinning effect of second phase particles and the frequency distribution of grain diameters. Based on these results, the influence of temperature in the Monte Carlo method has been discussed. The influence of temperature could be introduced in the Monte Carlo simulation through the correspondent relationship between the Monte Carlo Step and real time, because the influence of temperature could not be explained through the transition probability in re-orientation attempts. A new equation that represents the relationship between $M C S / Q$ and real time has been derived based on the idea of the law of similitude in grain growth. The grain growth during cooling for the real time-length system has been estimated using a newly derived equation for the relationship between $M C S / Q$ and temperature during cooling and the calculated relationship between the grain diameter and temperature has been compared with Yoshida et al.'s equation as an alternative for Maehara's observed results. ${ }^{15)}$ It has been revealed that calculated grain diameters in relation to temperature and cooling rate were quantitatively in good agreement with those calculated by Yoshida et al.'s equation when the lattice constant in the calculation system as a characteristic length for conversion from the calculation system to the real system was set to be $0.015 \mathrm{~cm}$ which was half of the initial grain diameter in the real system.

\section{REFERENCES}

1) M. P. Anderson, D. J. Srolovitz, G. S. Grest and P. S. Sahni: Acta Metall., 32 (1984), 783.

2) D. J. Srolovitz, M. P. Anderson, P. S. Sahni and G. S. Grest: Acta Metall., 32 (1984), 793.

3) J. A. Spittle and S. G. R. Brown: Acta Metall., 37 (1989), 1803.

4) E. Enomoto, M. Kamiya and T. Nagai: Mater. Sci. Forum, 204-206 (1996), 71.

5) D. Weygand, Y. Brechet and J. Lepinoux: Acta Mater., 47 (1999), 961.

6) D. Fan and L. Q. Chen: Acta Mater, 45 (1997), 611.

7) G. S. Grest, D. J. Srolovitz and M. P. Anderson: Acta Metall., 33 
ISIJ International, Vol. 48 (2008), No. 3

(1985), 509.

8) Y. Saito and M. Enomoto: ISIJ Int., 32 (1992), 267.

9) B. Radhakrishnan and T. Zacharia: Metall. Mater. Trans. A, 26A (1995), 167.

10) M. Kobayashi, Y. Takayama, H. Kato and H. Nakajima: J. Jpn. Inst. Met., 65 (2001), 85.

11) D. J. Srolovitz, M. P. Anderson and G. S. Grest: Acta Metall., 32 (1984), 1429

12) J. Gao, R. G. Thompson and B. R. Patterson: Acta Mater., 45 (1997) 3653 .
13) I. Ohnuma, H. Ohtani, K. Ishida and T. Nishizawa: J. Jpn. Inst. Met., 58 (1994), 1133.

14) N. Yoshida, Y. Kobayashi and K. Nagai: Tetsu-to-Hagané, 90 (2004), 198.

15) Y. Maehara, K. Yasumoto, Y. Sugitani and K. Gunji: Trans. Iron Steel Inst. Jpn., 25 (1985), 1045.

16) J. Gao and R. G. Thompson: Acta Mater., 44 (1996), 4565.

17) T. Matsumiya: Materia Jpn., 35 (1996), 912.

18) N. Yoshida, O. Umezawa and K. Nagai: ISIJ Int., 44 (2004), 547. 